\title{
Thermodynamic and numerical analysis of intake air humidification of a turbocharged GDI engine
}

\author{
${\text { BIN } \mathrm{CHEN}^{1} \text {, LI ZHANG }}^{1, * \odot}, \mathrm{XI} \mathrm{CHEN}^{1}$ and QING ZHANG ${ }^{2}$ \\ ${ }^{1}$ School of Automotive Engineering, Chongqing University, Chongqing 400044, People's Republic of China \\ ${ }^{2}$ Changan Automobile Company Limited, Chongqing 400044, People's Republic of China \\ e-mail: $20153201011 @$ cqu.edu.cn
}

MS received 2 September 2017; revised 29 January 2018; accepted 11 March 2018; published online 14 May 2018

\begin{abstract}
Reducing the temperature and increasing the specific heat capacity of working medium of gasoline engines are the most efficient methods of mitigating knock tendency. The charge cooling effect of intake air humidification is helpful for decreasing the initial temperature of intake air, and the increase of the specific heat capacity of working medium can reduce the temperature rise in the in-cylinder process. This study established a mathematical model of intake air humidification of gasoline engines, and analyzed the effects of the technique on the thermodynamic process of a turbocharged gasoline engine with Ricardo WAVE Code. The results indicated that the intake air humidification is an isenthalpic process; the vapor influences the working process of the engine by altering the thermodynamic parameters of the working medium. A decrease in the initial temperature and adiabatic index and an increase in the specific heat capacity of working medium lowered the incylinder temperature and pressure, hence suppressing the knock occurrence. After the humidification of intake air, the engine performance slightly increased, and the thermal efficiency showed different levels of improvements at all the working conditions.
\end{abstract}

Keywords. Intake air humidification; knock; gasoline engine; charge; thermodynamics; water injection.

\section{Introduction}

With the development of society, environmental problems have attracted much attention; more stringent vehicle emission regulations have been introduced. Coupled with the rapid development of electric vehicles in recent years, internal combustion engines adopted diverse new technologies to improve the power output and thermal efficiency while reducing emissions to meet the market requirements. For gasoline engines, the application of charging technology is undoubtedly a landmark. However, intake boosting increases the heat load and worsens the knock tendency of gasoline engines. Therefore, the compression ratio is reduced, and the mixture is enriched, in order to reduce the knock tendency in practice. This is detrimental to the improvement of thermal efficiency and fuel economy. As a result, knock became one of the main obstacles in the development of gasoline engines.

Douaud and Eyzat [1] studied knock and reported that the temperature of unburned mixture plays a significant role in knock occurrence. Therefore, the reduction of the mixture temperature of a gasoline engine has been an important topic in knock researches. At present, the most frequently used method for reducing the temperature of mixture is

*For correspondence intercooling. However, in consequence of the mounting position, the size of intercooler should be as small as possible, that result in a reduction in the cooling effect. Studies in [2, 3] indicate that an increase in the adiabatic index of mixture decreased the mixture temperature, thus suppressing knock. Cooled exhaust gas recirculation (EGR), which is widely applied nowadays, is the major technique of achieving this purpose. However, the structure of cooled EGR system is complex and it must be precisely controlled. Intake air humidification combining advantages of the two aforementioned techniques was first applied to aeroengines in World War II [5, 6], but for automotive gasoline engines, the technology is still in the initial stage. Even though intake air humidification cannot completely replace intercooler, but it indeed maximizes charge cooling effect, and its structure is simple in comparison with cooled EGR. Due to it works only at limit engine operations, the consumption of water is tiny. According to current studies, the benefit gained from intake air humidification is greater than the negative impact.

Intake air humidification significantly reduces the temperature of intake air as well as improves the adiabatic index because of the higher latent heat of vaporization and specific heat capacity of water. Current studies indicate that intake air humidification reduces emissions [7-15], improves power output and fuel economy [7, 9-11, 13, 16], as well as mitigates knock tendency [4, 16-18]. 
The current studies emphasized on performance tests and simulations, while there are few theoretical analyses on the changes in the thermodynamic process of gasoline engines and the effects of intake air humidification on knock. In this case, this study established a thermodynamic model for the intake air humidification of a gasoline engine, mathematically and numerically analyzed the effects of water addition on the working medium in the working process and knock occurrence of gasoline engines.

\section{Research methods}

The intake air was considered as dry air in this study. The mass of air aspirated per cycle did not vary with the addition of water. The airflow in the intake duct was an adiabatic steady flow, and all the heat required for the evaporation of added water was provided by the dry air in the intake duct.

There are two methods for adding water to the intake air of engines: (i) injecting water into the intake duct and (ii) directly injecting water into the cylinder. The first method was employed in this study, as shown in figure 1 . The advantage of injecting water to the intake duct is that the injected water has sufficient time to evaporate, it is prevented from entering the cylinder to destroy the oil film and causing mechanical damages to the engine.

The effect of intake air humidification of gasolines on knock tendency was analyzed based on the induction-time equation originating from Arrhenius equation:

$$
1=\int_{t_{0}}^{t_{c}} \frac{1}{\tau} d t
$$

where $t_{0}$ is the start of the compression stroke; $t_{c}$ is the time when the integral value reaches 1 , the time of knock occurrence. The Induction-time $\tau$ was calculated from the most frequently used Douaud and Eyzat [1] equation in knock analysis:

$$
\tau=18.33\left(\frac{O N}{100}\right)^{3.402} p^{-1.7} \exp \left(\frac{3800}{T}\right)
$$

where $O N$ is the fuel octane Number, $p$ is the instantaneous cylinder pressure, $T$ is the instantaneous unburned gas temperature. Equation (2) shows that induction-time $\tau$ is the function of instantaneous cylinder temperature, pressure, and fuel octane number.

\section{Establishment of numerical model}

\subsection{Intake air humidification}

The air in intake duct was discretized into separate air units based on the mass of air inhaled per cycle in the thermodynamic analysis, as shown in figure 2 . According to the energy conservation law, the energy equation of the air flowing past the air unit can be expressed as follows:

$$
\delta Q=d H+\frac{1}{2} m d c_{f}^{2}+m g d_{z}+\delta W_{s}
$$

As the intake air humidification is an adiabatic process without energy exchange and the Macro kinetic and potential energies of intake air are ignored in the adiabatic process, the energy-balance equation can be expressed as follows:

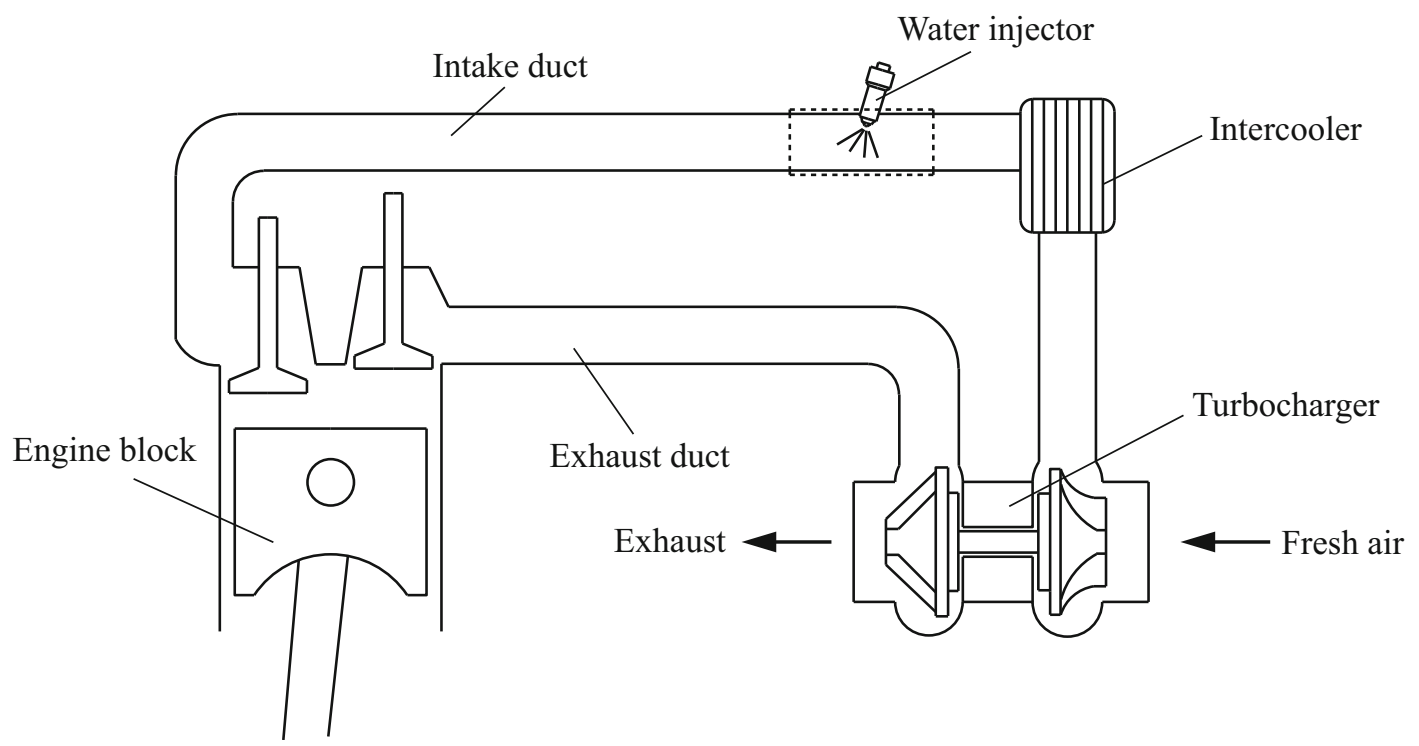

Figure 1. Engine layout with water injector. 


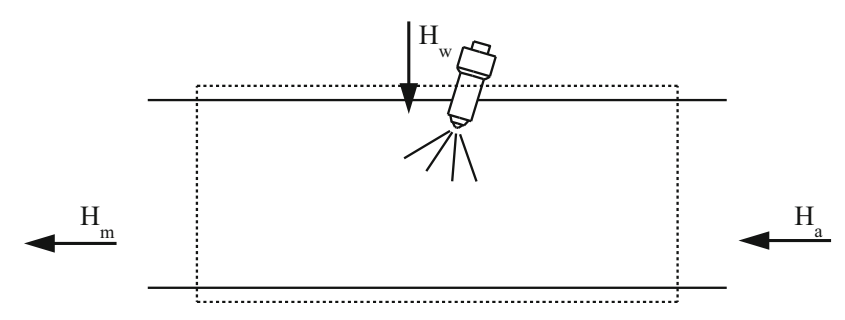

Figure 2. The micro unit of intake air.

$$
H_{m}=H_{a}+H_{w}
$$

where $H_{m}$ is the enthalpy of the humidified intake air, $H_{a}$ is the enthalpy of the dry air entering the air unit, $H_{w}$ is the enthalpy of the water added to the air unit. Equation (4) indicates that the humidification is an isenthalpic process. The added water absorbs heat at the time of evaporation, and the air temperature decreases. The decrease in sensible heat of air is equal to the increase in the latent heat of vapor. The total enthalpy of air unit remains constant during the humidification.

The humidified intake air temperature can be calculated using equation (5) as follows:

$$
\begin{aligned}
& m_{a} c_{p a} T_{a}+m_{w} h_{w}=m_{a} c_{p a} T_{m}+m_{v} h_{c}+m_{v} c_{p v} T_{m} \\
& T_{m}=\frac{m_{a} c_{p a} T_{a}+m_{w} h_{w}-m_{v} h_{c}}{m_{a} c_{p a}+m_{v} c_{p v}} m_{a}
\end{aligned}
$$

where $m_{a}$ is the mass of the dry air entering the air unit, $m_{w}$ is the mass of the water injected into the air unit, $m_{v}$ is the mass of the vapor, $c_{p a}$ is the specific heat capacity at constant pressure, $T_{a}$ is the dry air temperature, $h_{w}$ is the specific enthalpy of water, $h_{c}$ is the enthalpy of saturated steam at $0{ }^{\circ} \mathrm{C}, c_{p v}$ is the specific heat capacity at constant pressure, $T_{m}$ is the humidified intake air temperature.

Because the mass of air aspirated per cycle was assumed to be constant, the effects of pressure drop on the capacity of intake duct is constant. The humidification is a constantvolume process; therefore, the partial pressure of dry air in the humidified air can be calculated using equation (6):

$$
p_{m a}=p_{a} \frac{T_{m}}{T_{a}}
$$

The partial pressure of vapor in the humidified air is:

$$
p_{m v}=\phi p_{s}=\frac{m_{v}}{d_{\max }} p_{s}
$$

Therefore, the total pressure of the humidified intake air is:

$$
p_{m}=p_{m a}+p_{m v}
$$

where $d_{\max }$ is the maximum moisture content, $P_{s}$ is the partial pressure of the saturated moist air.

The maximum moisture content $d_{\max }$ was confirmed by determining the mass of dry air entering the air unit. Under ideal condition, when water mass $m_{w} \leq d_{\max }$, the added water exists as vapor, and the effect of water on the engine working process is confined within the intake duct. When $m_{w}>d_{\max }$, the added water exists in the state of both vapor and liquid, and the mass of liquid water is $m_{w}-d_{\max }$. This has a certain impact on the engine working process. Therefore, the maximum saturated content should be considered instead of determining the water mass merely according to the water-to-fuel ratio $[7,11,12,18]$ or the mass of injected water per hour when a theoretical analysis or bench test is carried out, or false results can be produced and water can have a negative effect on the engine and test equipment if water enters the cylinder.

\subsection{Thermodynamic analysis of in-cylinder process}

The effect of intake air humidification on gasoline engines was generally considered in two aspects: chemical reaction and thermodynamic process. In fact, water is a quite stable compound, and its formation heat is very large. Therefore, the probability of water to dissociate is tiny. As shown in table 1, the dissociation rate is around $2.94 \%$ at $2400 \mathrm{~K}$. Because the dissociation reaction is reversible, the absorbed heat will be released with the progress in reverse reaction when the temperature decreases. Therefore, water has a minor influence on gasoline engines resulting from dissociation. This study ignores the dissociation of water while emphasizing the changes in thermodynamic cycle caused by water.

The actual thermodynamic process of a gasoline engine was simplified to be a closed thermodynamic cycle in this paper. Combustion and heat release were considered as the system is heated by environment. Expansion was assumed as releasing heat to environment. Valves were opened and closed at the time of top and bottom dead centers. At the same time working medium entered or discharged from the cylinder instantaneously. The throttling loss and heat transfer were ignored. Figure 3 shows the simplified thermodynamic process of the gasoline engine, where 1-2 is adiabatic compression, $2-3$ is the addition of heat at a constant volume, 3-4 is adiabatic expansion, 4-1 is the rejection of heat at a constant volume.

Table 1. Thermal dissociation property of water.

\begin{tabular}{lcccccrrr}
\hline Temperature $(\mathrm{K})$ & 1000 & 1200 & 1400 & 1600 & 1800 & 2000 & 2200 & 2400 \\
\hline Dissociation rate $(\%)$ & 0.00003 & 0.00081 & 0.00681 & 0.051 & 0.199 & 0.588 & 1.42 & 2.94 \\
\hline
\end{tabular}



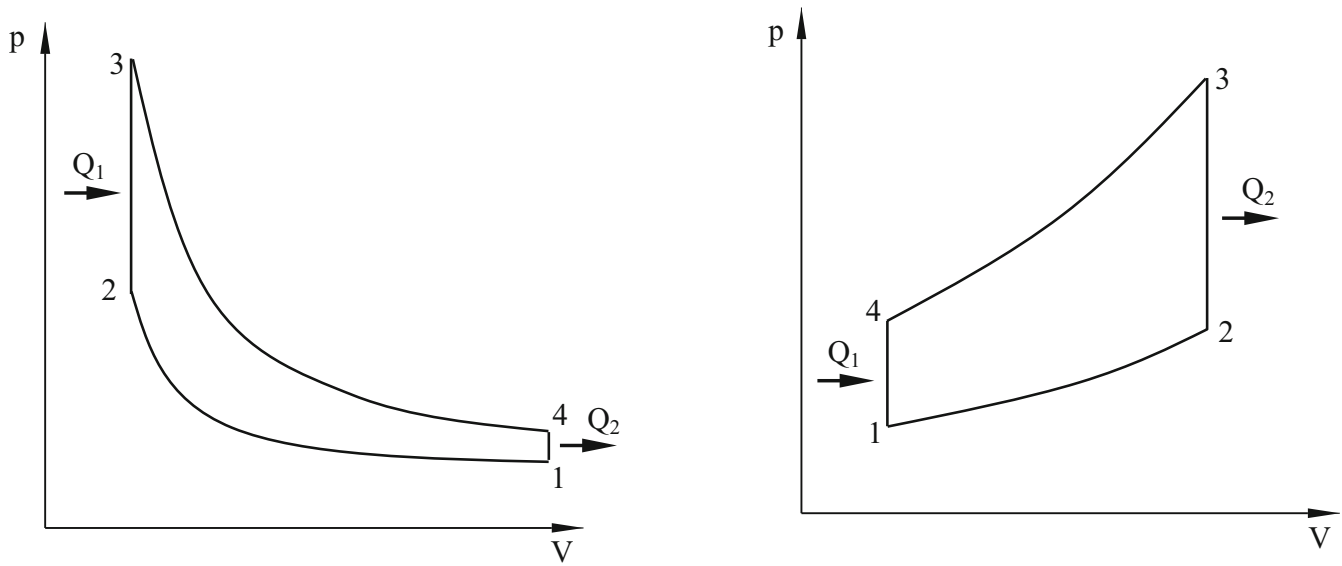

Figure 3. The $\mathrm{p}-\mathrm{V}$ diagram and $\mathrm{T}-\mathrm{S}$ diagram of constant volume heating.

Because throttling loss and heat transfer were ignored, the humidified intake air in the intake duct entered the cylinder by keeping the temperature and pressure constant. The state 1 in figure 3 is the initial state of working medium in the cylinder; its temperature and pressure are identical with those in the intake duct, i.e.,

$$
\begin{aligned}
& T_{1}=T_{m} \\
& p_{1}=p_{m}
\end{aligned}
$$

The temperature and pressure of the compressed working medium adiabatically at state 2 can be calculated from the initial-state parameters,

$$
\begin{gathered}
T_{2}=T_{1} \varepsilon^{\kappa_{2}^{1}-1} \\
p_{2}=p_{1} \varepsilon^{\kappa_{2}^{1}}
\end{gathered}
$$

where $\varepsilon$ is the compression ratio, and $\kappa_{2}^{1}$ is the adiabatic index in the adiabatic compression. The initial temperature and pressure of working medium entering cylinder decreased after the humidification, and the adiabatic index also changed. It can be deduced from the specific heat capacity equation of mixture, $c=\sum_{i} \omega_{i} c_{i}$, that the adiabatic index of humidified working medium is

$$
\begin{aligned}
\kappa_{2}^{1} & =\frac{c_{p 2}^{1}}{c_{v 2}^{1}}=\frac{\left(m_{a} c_{p a 2}^{1}+m_{v} c_{p v 2}^{1}\right) /\left(m_{a}+m_{v}\right)}{\left(m_{a} c_{v a 2}^{1}+m_{v} c_{v v 2}^{1}\right) /\left(m_{a}+m_{v}\right)} \\
& =\frac{\left(m_{a} c_{p a 2}^{1}+m_{v} c_{p v 2}^{1}\right)}{\left(m_{a} c_{v a 2}^{1}+m_{v} c_{v v 2}^{1}\right)}
\end{aligned}
$$

where $c_{p 2}^{1}, c_{p a 2}^{1}$, and $c_{p v 2}^{1}$ are the specific heat capacity of mixture, dry air, and vapor at a constant pressure in the adiabatic compression, respectively, $c_{v 2}^{1}, c_{v a 2}^{1}$, and $c_{v v 2}^{1}$ are the specific heat of mixture, dry air, and vapor at a constant volume, respectively. Equation (13) indicates that the specific heat capacity of mixture increased and the adiabatic index decreased with humidification. This is because the specific heat capacity of vapor is larger than that of air, and the variation increases with the increase in humidification. Equation (12) shows that the temperature and pressure at the end of compression stroke decreased with the decrease in adiabatic index.

The heat added to the system during the addition of heat marked as 2-3 in figure 3 is

$$
Q_{1}=g_{b} H_{u} \eta_{c}
$$

where $g_{b}$ is the cycle fuel injection quantity, $H_{u}$ is the lower heating value of fuel, and $\eta_{c}$ is the combustion efficiency. The heat added to the system was absorbed completely by the working medium; therefore,

$$
Q_{1}=Q_{a s}+Q_{v s}
$$

$Q_{a s}$ and $Q_{v s}$ are the heat absorbed by dry air and vapor, expressed separately as follows:

$$
\begin{aligned}
& Q_{a s}=m_{a} c_{v a 3}^{2}\left(T_{3}-T_{2}\right) \\
& Q_{v s}=m_{v} c_{v v 3}^{2}\left(T_{3}-T_{2}\right)
\end{aligned}
$$

The temperature and pressure of the working medium at state 3 can be calculated using Equations (18) and (19),

$$
\begin{gathered}
T_{3}=\frac{g_{b} H_{u} \eta_{c}}{m_{a} c_{v a 3}^{2}+m_{v} c_{v v 3}^{2}}+T_{2} \\
p_{3}=p_{2} \frac{T_{3}}{T_{2}}
\end{gathered}
$$


where $c_{v a 3}^{2}$ and $c_{v v 3}^{2}$ are the specific heat capacity at a constant volume of air and vapor between states 2 and 3 .

Equations (18) and (19) show that the higher specific heat capacity of water makes it absorb more heat, thus decreasing the temperature and pressure of working medium between the start of compression and ignition timing. The decrease in temperature and pressure increase induction time $\tau$ directly, hindering knock occurrence.

The temperature $T_{4}$, pressure $p_{4}$, and adiabatic index $\kappa_{4}^{3}$ at state 4 are expressed as follows:

$$
\begin{gathered}
T_{4}=T_{3}\left(\frac{1}{\varepsilon}\right)^{\kappa_{4}^{3}-1} \\
p_{4}=p_{3}\left(\frac{1}{\varepsilon}\right)^{\kappa_{4}^{3}} \\
\kappa_{4}^{3}=\frac{c_{p 4}^{3}}{c_{v 4}^{3}}=\frac{\left(m_{a} c_{p a 4}^{3}+m_{v} c_{p v 4}^{3}\right)}{\left(m_{a} c_{v a 4}^{3}+m_{v} c_{v v 4}^{3}\right)}
\end{gathered}
$$

where $c_{p a 4}^{3}$ and $c_{p v 4}^{3}$ are the specific heat capacity at a constant pressure of air and vapor between states 3 and 4, $c_{v a 4}^{3}$ and $c_{v v 4}^{3}$ are the specific heat capacity at a constant volume of air and vapor between states 3 and 4 .

The heat released during the rejection at a constant volume can be expressed as follows:

$$
Q_{2}=Q_{a e}+Q_{v e}=\left(m_{a} c_{v a 1}^{4}+m_{v} c_{v v 1}^{4}\right)\left(T_{4}-T_{1}\right)
$$

where $Q_{a e}$ and $Q_{v e}$ are the heat released from dry air and vapor; $c_{v a 1}^{4}$ and $c_{v v 1}^{4}$ are the specific heat capacity at a constant volume of air and vapor between states 4 and 1 .

The thermal efficiency of gasoline engine was calculated from $Q_{1}$ and $Q_{2}$ using the following equation:

$$
\eta_{t}=1-\frac{Q_{2}}{Q_{1}}=1-\frac{\left(m_{a} c_{v a 1}^{4}+m_{v} c_{v v 1}^{4}\right)\left(T_{4}-T_{1}\right)}{\left(m_{a} c_{v a 3}^{2}+m_{v} c_{v v 3}^{2}\right)\left(T_{3}-T_{2}\right)}
$$

The thermodynamic equations mentioned above underline that the temperature and pressure of working medium decreased after the humidification. This is the main reason of intake air humidification suppressed knock occurrence.

\subsection{Numerical analysis of the intake air humidification of a gasoline engine}

The base engine is a $2.0 \mathrm{~L}$ turbocharged gasoline engine with direct injection, the main parameters of which are given in table 2 . The numerical analysis was carried out using the GT-Power Code. The predicted data of the model were compared with the measured data to verify the precision of the engine model. It can be observed from figure 4 that the predicted data of the engine model are in line with the measured data, the maximum deviation of power,
Table 2. Main parameters of the base engine.

\begin{tabular}{lc}
\hline Type & $\begin{array}{c}\text { Turbocharged Gasoline direct injection, 4 } \\
\text { strokes, } 4 \text { cylinders }\end{array}$ \\
\hline Bore/Stroke & $86 / 86.07 \mathrm{~mm}$ \\
$\begin{array}{l}\text { Displacement } \\
\text { Connecting rod } \\
\text { length }\end{array}$ & $2.0 \mathrm{~L}$ \\
$\begin{array}{l}\text { Compression } \\
\text { ratio }\end{array}$ & $175 \mathrm{~mm}$ \\
\hline
\end{tabular}

torque, BSFC, and air flow are $2.6 \%, 2.2 \%, 1.3 \%$, and $1.4 \%$, respectively, which means that the model is rational enough to carry out the study of intake air humidification.

VDO knock diagnosis method was used in this study to detect knock occurrence in the knock test. Because of the randomness of knock, the knock intensity $\mathrm{KH}$ was measured from the percentage of knock cycles among 200 cycles. $\mathrm{KH}$ less than $1 \%$ means no knock occurs; $\mathrm{KH}$ between $1 \%$ and $5 \%$ indicates knock threshold; $\mathrm{KH}$ between $5 \%$ and $10 \%$ means light knock; $\mathrm{KH}$ greater than $10 \%$ means severe knock. The tested knock threshold of the base engine presented in figure 5 shows that the base engine exhibits knock phenomenon at high load operations and all speeds.

In the numerical study, the Douaud and Eyzat knock model was employed to assess knock. Knock Index is a value based on time defined as follows:

$$
\begin{array}{r}
K I=10000 M u \frac{V_{T D C}}{V} \exp \left(\frac{-6000}{T}\right) \max \left(0,1-(1-\Phi)^{2}\right) \\
\frac{I_{\text {ave }}}{I_{K-\text { ref }} I_{K-\text { corr }}}
\end{array}
$$

where $M$ is the knock index multiplier, $u$ is the percentage of cylinder mass unburned, $V_{T D C}$ is the cylinder volume at the top dead center, $V$ is the cylinder volume, $T$ is the bulk unburned gas temperature $(\mathrm{K}), \Phi$ is the equivalence ratio of the unburned zone, $I_{\text {ave }}$ is the induction time integral, $\mathrm{I}_{\mathrm{K} \text {-ref }}$ is the reference induction time integral, $I_{K \text {-corr }}$ is the induction time integral correlation factor.

The water injection module was located downstream the intercooler and injected water into the intake duct. The cyclic water injection quantity was controlled by setting the injection rate or injection pulse-width and determined by the percentage of cycle fuel injection. The water-to-fuel ratios studied were $0.1,0.2,0.3$, and 0.4 .

\section{Results and analysis}

Figure 6 shows the in-cylinder temperature behavior at $3000 \mathrm{rpm}$ and full load with different water-to-fuel ratios. Figure 7 shows the temperature change after water was 

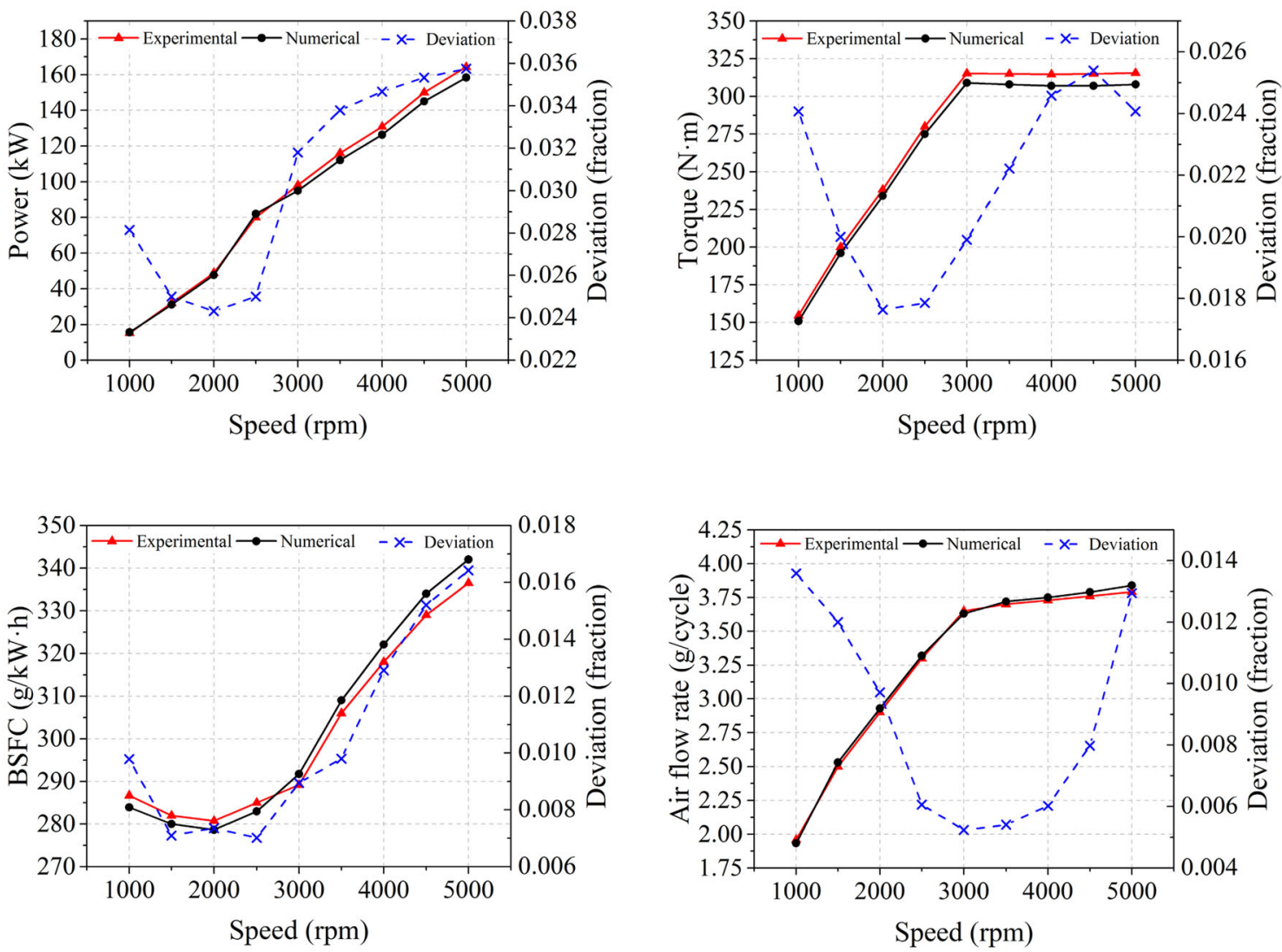

Figure 4. Comparison of measured and predicted data.

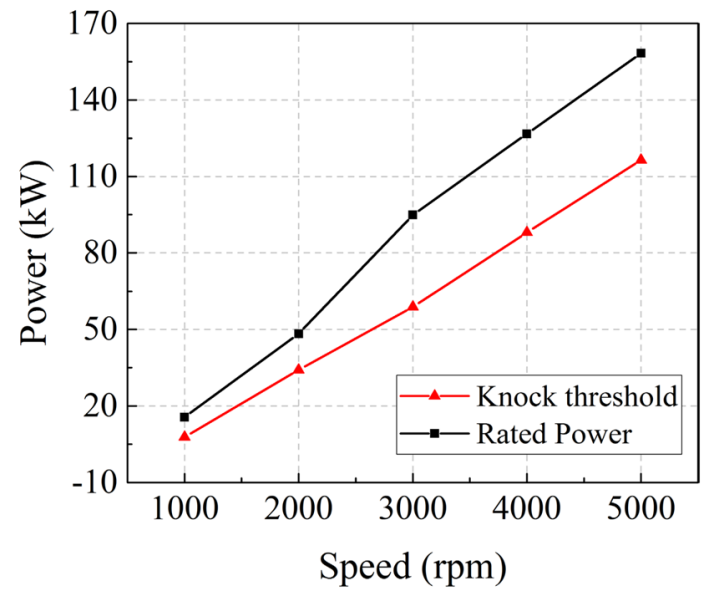

Figure 5. Knock boundary of the based engine.

added to intake air compared to the original situation at $3000 \mathrm{rpm}$ and full load. The temperature change $\Delta T$ is defined as follows:

$$
\Delta T=T_{0}-T_{R_{w / f}}
$$

where $T_{0}$ is the in-cylinder temperature of the base engine, $T_{R_{w / f}}$ is the in-cylinder temperature after water was added to intake air.

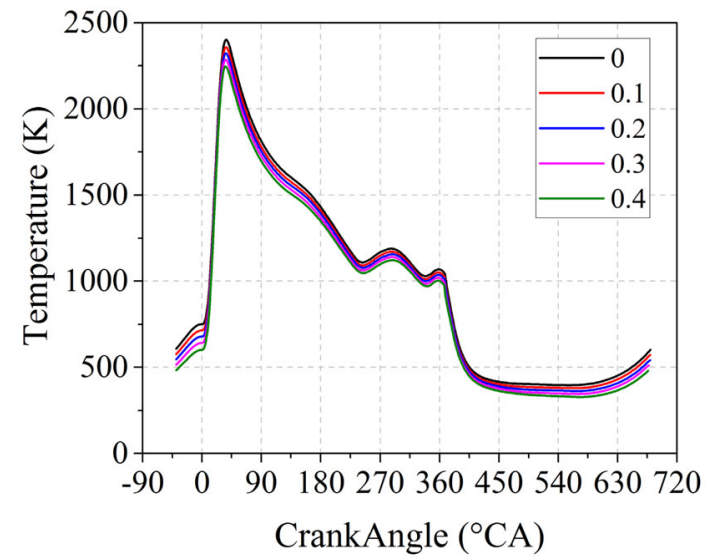

Figure 6. In-cylinder temperature curves (3000 rpm, full load).

It indicates that by combining figures 6 and 7, the incylinder temperature decreases with the increase in water addition quantity. The intake air humidification had the largest influence on the initial temperature of working medium, and the influence decreased with the progress in intake stroke. This is because water has a larger latent heat of vaporization, and the water injected into the intake duct absorbs a large amount of heat to vaporize, leading to a 


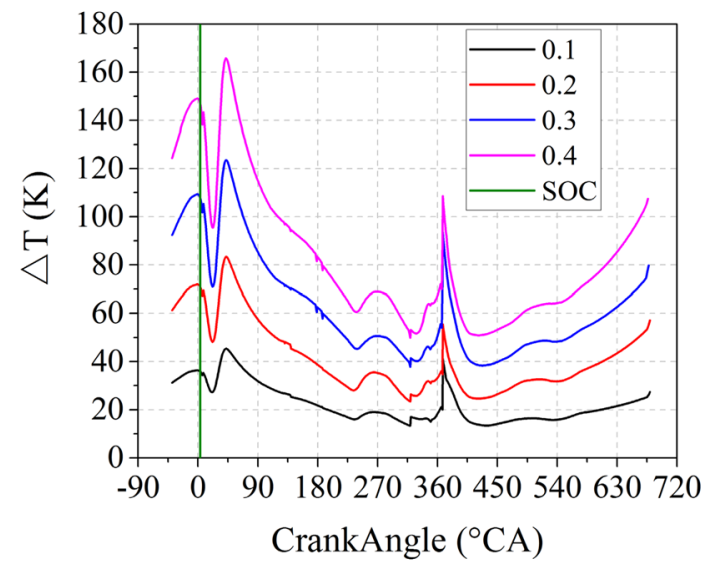

Figure 7. Reduction of in-cylinder temperature (3000 rpm, full load).

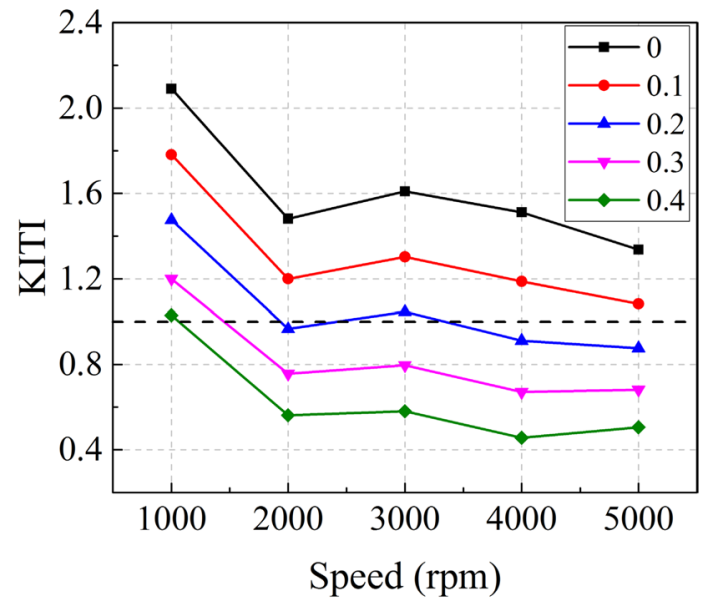

Figure 8. Knock induction time.

sharp decrease in the initial temperature of the working medium. With the progress in compression, the decrease in in-cylinder temperature increased and achieved its peak value at the top dead center. This is because the addition of water increases the specific heat capacity and decreases the adiabatic index of a working medium. A sharp increase in the in-cylinder temperature was observed after the start of combustion (SOC), but the effect of water on in-cylinder temperature decreased. This is because the change in the initial combustion temperature is not so large that it can significantly affect the combustion temperature. The incylinder peak temperature clearly decreased to $42.92 \mathrm{~K}$, $78.14 \mathrm{~K}, 116.02 \mathrm{~K}$, and $156.18 \mathrm{~K}$ with water-to-fuel ratios of $0.1,0.2,0.3$, and 0.4 , respectively. The in-cylinder temperature and its change gradually decreases with the progress in expansion stroke until the end of the exhaust stroke. The decrease in the in-cylinder temperature lead to a decrease in the induction time integral, benefiting the suppression of knock.

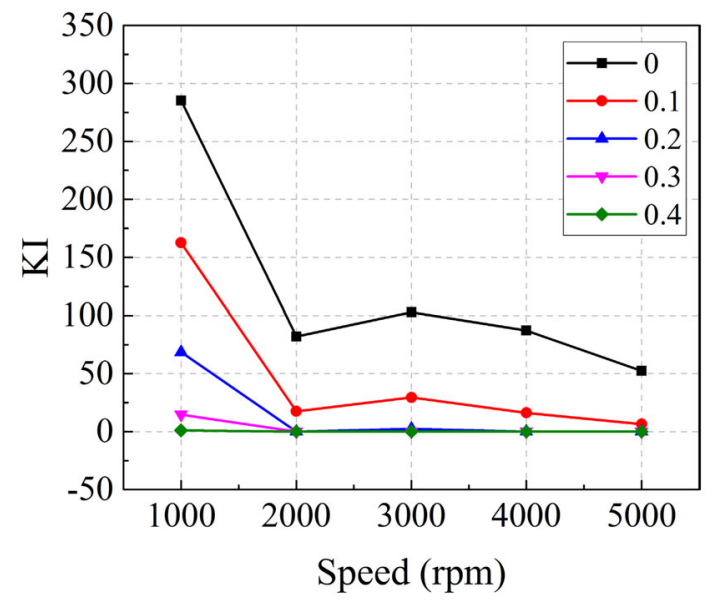

Figure 9. Knock index.

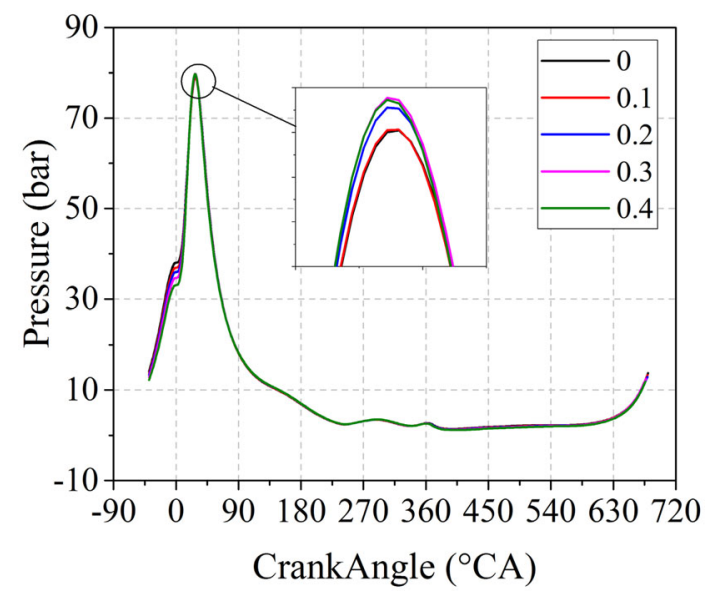

Figure 10. In-cylinder pressure curves (3000 rpm, full load).

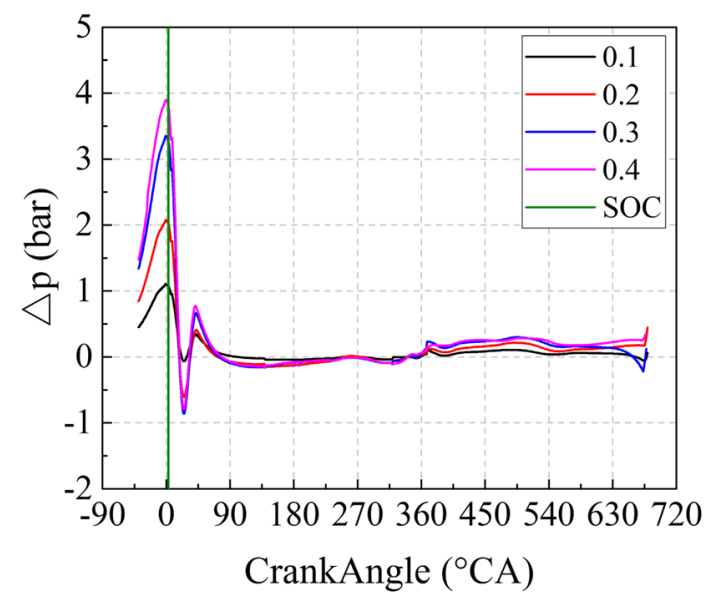

Figure 11. Reduction of in-cylinder pressure (3000 rpm, full load). 


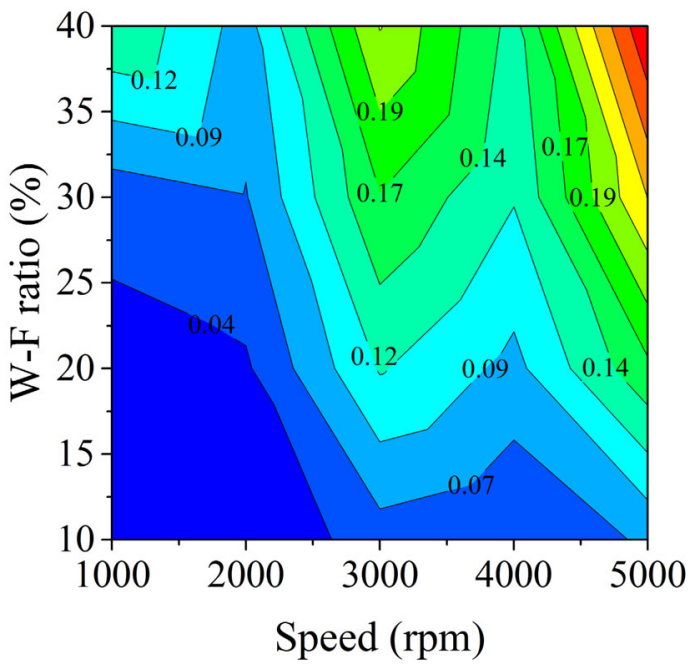

Figure 12. Increase of air flow (g/cycle).

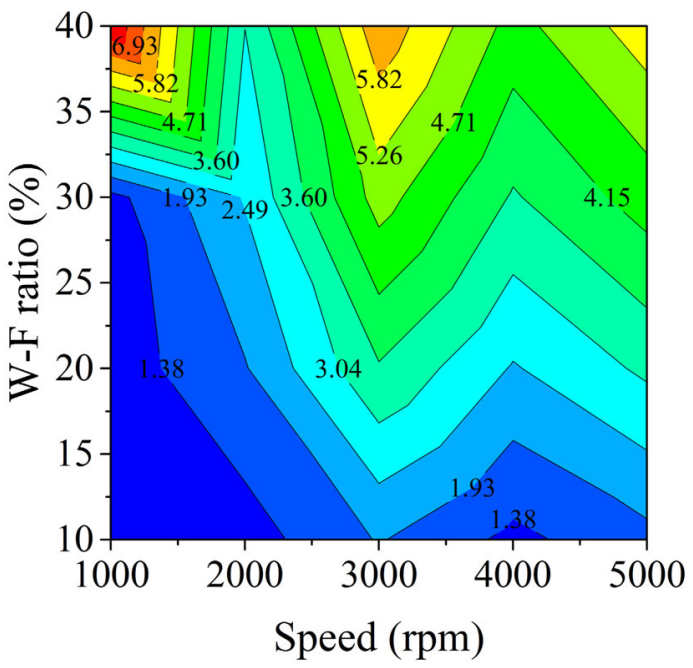

Figure 13. Increase of torque output $(\mathrm{N} \cdot \mathrm{m})$.

As shown in figure 8 , the induction time integral significantly decreased with the increase in water-to-fuel ratio. The dashed line in figure 8 shows the knock threshold. Figures 8 and 9 show that the engine knock was completely suppressed when the water-to-fuel ratios were $0.4,0.2,0.3$, 0.2, and 0.2 at $1000 \mathrm{rpm}, 2000 \mathrm{rpm}, 3000 \mathrm{rpm}, 4000 \mathrm{rpm}$, and $5000 \mathrm{rpm}$, respectively.

Figure 10 shows the in-cylinder pressure behavior with different water-to-fuel ratios at $3000 \mathrm{rpm}$, and figure 11 shows the pressure change after water was added to the intake air compared to the original situation at $3000 \mathrm{rpm}$. The pressure change $\Delta p$ is defined as follows:

$$
\Delta p=p_{0}-p_{R_{w / f}}
$$

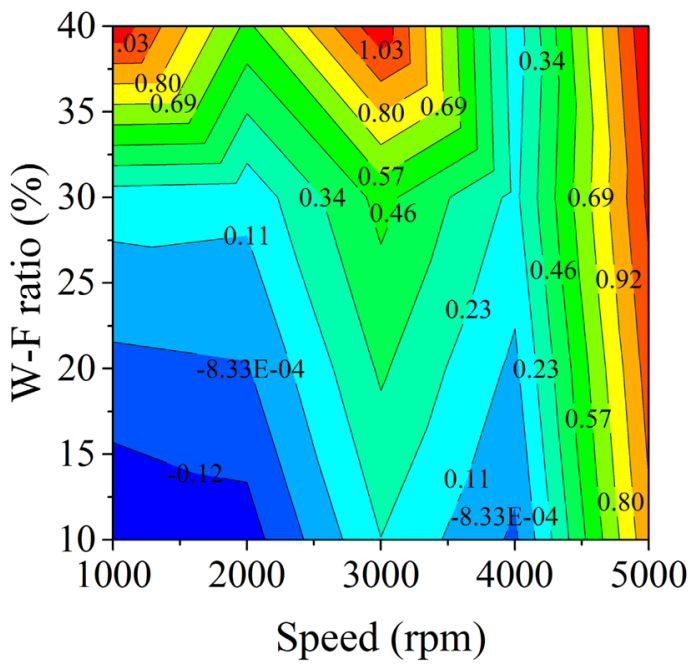

Figure 14. Increase of brake thermal efficiency (\%).

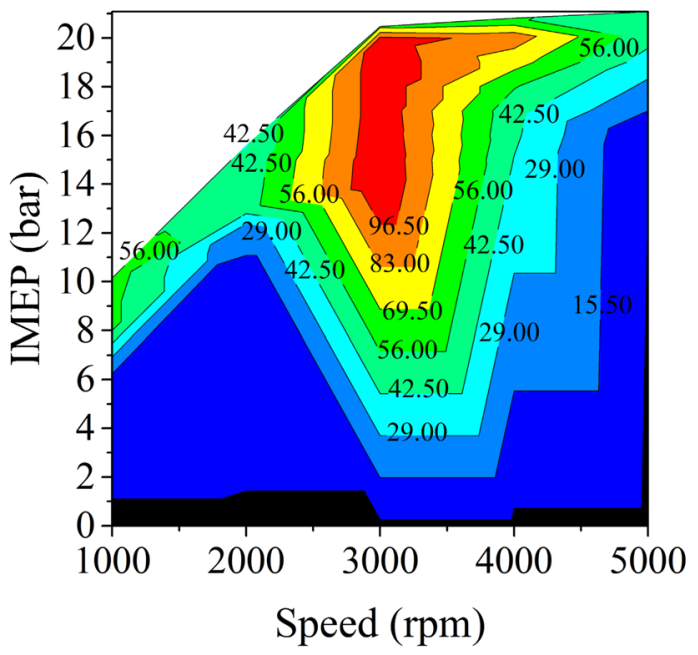

Figure 15. Injected water at all working conditions (mg/cycle).

where $p_{0}$ is the in-cylinder pressure of the original engine, $p_{R_{w / f}}$ is the in-cylinder temperature after water was added to intake air.

Figures 10 and 11 show that the in-cylinder pressure between the start of intake stroke and ignition timing clearly decreased. Especially at the top dead center, the pressure reduction was 3.89 bar when the water-to-fuel ratio was 0.4 . The pressure decrease became negative after the ignition. This indicates that the in-cylinder pressure increased, and the largest pressure increment was 0.79 bar the when water-to-fuel ratio was 0.3 . The in-cylinder pressure showed a slight change in the expansion stroke, even though the pressure clearly decreased at the top dead center. This is because the vapor is an inert gas under the in-cylinder condition; therefore, the increase in water-to-fuel ratio means that there are more working media in cylinder. 
The mass of intake air increased with the decrease in temperature. As shown in figure 12, the mass of cycle intake air increased with the increase in water-to-fuel ratio, and the most obvious increment occurred at high speed operations. The largest increment was $8.837 \%$ at $5000 \mathrm{rpm}$ when the water-to-fuel ratio was 0.4 .

The increase in cycle intake air mass improved the torque output, as shown in figure 13. The largest increment of torque was $7.46 \mathrm{~N} \cdot \mathrm{m}$ at $1000 \mathrm{rpm}$ when the water-to-fuel ratio was 0.4 .

Figure 14 shows that the thermal efficiency increased at all the operations. As shown in figure 15, the mass of water injection increased with the increase in loads because of the high knock tendency.

\section{Conclusions}

It can be inferred from the study that the added water mainly affects the thermodynamic process of gasoline engines but the effects of chemical reaction is tiny. The thermodynamic equations of intake air humidification of gasoline engines indicate that the process of humidification is an isenthalpic process. The in-cylinder temperature and pressure decrease because of both the increase in specific heat capacity and decrease in the adiabatic index of working medium. Therefore, intake air humidification is an efficient strategy of suppressing knock occurrence of gasoline engines.

Although it needs to be further confirmed by experiment, but this study illuminated the principles of how the technique affects the working process of gasoline engines with mathematical analysis, and validated its effects with a numerical model, hence provides a good guideline for practice.

\section{Acknowledgement}

This research is supported by Specialized Research Fund for Key Industry of Chongqing, China (Grant No. cstc2015zdcy-ztzx60014). Government of Chongqing and Changan Automobile Company are gratefully acknowledged.

\section{Nomenclature}

EGR exhaust gas recirculation

$t_{a} \quad$ temperature of dry air

$t_{0} \quad$ start of the compression stroke

$t_{c} \quad$ time of knock occurrence

$\tau \quad$ induction-time

ON fuel octane Number

$p \quad$ instantaneous cylinder pressure

$p_{m a} \quad$ partial pressure of dry air

$p_{m v} \quad$ partial pressure of vapor in the humidified air

$p_{m} \quad$ total pressure of the humidified intake air $p_{0} \quad$ in-cylinder pressure of the original engine

$P_{s} \quad$ partial pressure of the saturated moist air

$p_{R_{w / f}} \quad$ in-cylinder temperature after water was added to intake air

$P_{1} \quad$ pressure at state 1

$P_{2} \quad$ pressure at state 2

$P_{3} \quad$ pressure at state 3

$P_{4} \quad$ pressure at state 4

$T$ instantaneous unburned gas temperature

$T_{a} \quad$ air temperature before humidification

$T_{m} \quad$ temperature of humidified intake air

$T_{1} \quad$ temperature at state 1

$T_{2} \quad$ temperature at state 2

$T_{3} \quad$ temperature at state 3

$T_{4} \quad$ temperature at state 4

$T_{0} \quad$ in-cylinder temperature of the base engine

$T_{R_{w / f}} \quad$ in-cylinder temperature after water was added to intake air

$H_{m} \quad$ enthalpy of the humidified intake air

$H_{a} \quad$ enthalpy of the dry air entering the air unit

$H_{w} \quad$ enthalpy of the water added to the air unit

$H_{u} \quad$ lower heating value of fuel

$m_{a} \quad$ mass of the dry air entering the air unit

$m_{w} \quad$ mass of the water injected into the air unit

$m_{v} \quad$ mass of the vapor

$c_{p a} \quad$ specific heat capacity at constant pressure

$c_{p v} \quad$ specific heat capacity at constant pressure

$c \quad$ specific heat capacity

$c_{p 2}^{1} \quad$ specific heat capacity of mixture at constant

pressure

$c_{p a 2}^{1} \quad$ specific heat capacity of dry air at constant pressure

$c_{p v 2}^{1} \quad$ specific heat capacity of vapor at constant pressure

$c_{v 2}^{1} \quad$ specific heat of mixture at constant volume between States 1 and 2

$c_{v a 2}^{1} \quad$ specific heat of dry air at constant volume between States 1 and 2

$c_{v v 2}^{1} \quad$ specific heat of vapor at constant volume between States 1 and 2

$c_{v a 3}^{2}$ specific heat of air at constant volume between States 2 and 3

$c_{v v 3}^{2}$

$c_{\text {pa4 }}^{3}$

$c_{p v 4}^{3}$

$c_{v a 4}^{3}$

$c_{v v 4}^{3}$

$c_{v a 1}^{4}$

$c_{v v 1}^{4}$ specific heat of vapor at constant volume between States 2 and 3

specific heat of air at constant pressure between States 3 and 4

specific heat of vapor at constant pressure between States 3 and 4

specific heat capacity of air at constant volume between States 3 and 4

specific heat capacity of vapor at constant volume between States 3 and 4

specific heat of air at constant volume between

States 4 and 1

specific heat of vapor at constant volume between States 4 and 1 
$h_{w} \quad$ specific enthalpy of water

$h_{c} \quad$ enthalpy of saturated steam at $0{ }^{\circ} \mathrm{C}$

$d_{\max }$ the maximum moisture content

$\varepsilon \quad$ compression ratio

$\kappa_{2}^{1} \quad$ adiabatic index in the adiabatic compression

$\kappa_{4}^{3} \quad$ adiabatic index between States 3 and 4

$Q_{1} \quad$ heat added to the system

$g_{b} \quad$ cycle fuel injection quantity

$\eta_{c} \quad$ combustion efficiency

$Q_{a s} \quad$ heat absorbed by dry air

$Q_{v s} \quad$ heat absorbed by vapor

$Q_{2} \quad$ heat released during the rejection at constant volume

$Q_{a e} \quad$ heat released from dry air

$Q_{v e} \quad$ heat released from vapor

$K I \quad$ knock index

$M \quad$ knock index multiplier

$u \quad$ percentage of cylinder mass unburned

$V_{T D C} \quad$ cylinder volume at the top dead center

$V \quad$ cylinder volume

$\Phi$ equivalence ratio of the unburned zone

$I_{\text {ave }} \quad$ induction time integral

$I_{K \text {-ref }} \quad$ reference induction time integral

$I_{K \text {-corr }}$ induction time integral correlation factor

$\Delta T \quad$ temperature change

$\Delta p \quad$ pressure change

\section{References}

[1] Douaud A and Eyzat P 1978 Four-Octane-Number Method for Predicting the Anti-Knock Behavior of Fuels and Engines. SAE Technical Paper 780080

[2] Hoepke B, Jannsen S, Kasseris E and Cheng W 2012 EGR Effects on Boosted SI Engine Operation and Knock Integral Correlation. SAE Int. J. Engines 2012-01-0707

[3] Francqueville L D and Michel J B 2014 On the effects of EGR on spark-ignited gasoline combustion at high load. SAE Int. J. Engines 2014-01-2628

[4] Bozza F, Bellis V D and Teodosio L 2016 Potentials of cooled egr and water injection for knock resistance and fuel consumption improvements of gasoline engines. Appl. Energy 169: 112-125

[5] Rothrock A M, Krsek A J and Jones A W 1943 The induction of water to the inlet air as a means of internal cooling in aircraft-engine cylinders. Technical Report Archive \& Image Library

[6] Miller C D 1944 End-zone water injection as a means of suppressing knock in a spark-ignition engine. Technical Report Archive \& Image Library

[7] Şahin Z, Tuti M and Durgun O 2014 Experimental investigation of the effects of water adding to the intake air on the engine performance and exhaust emissions in a DI automotive diesel engine. Fuel 115(115): 884-895

[8] Debertin D L and Pagoulatos A 2013 The effects of electronic controlled steam injection on spark ignition engine. Appl. Therm. Eng. 55(1-2): 61-68

[9] Wei M, Sa N T, Turkson R F, Liu J and Guo G 2016 Water injection for higher engine performance and lower emissions. J. Energy Inst. 90(2): 285-299

[10] Nande A M, Wallner T and Naber J 2008 Influence of water injection on performance and emissions of a direct-injection hydrogen research engine. SAE Technical Paper 2008-012377

[11] Adnan R, Masjuki H H and Mahlia T M I 2012 Performance and emission analysis of hydrogen fueled compression ignition engine with variable water injection timing. Energy 43(1): 416-426

[12] Tauzia X, Maiboom A and Shah S R 2010 Experimental study of inlet manifold water injection on combustion and emissions of an automotive direct injection Diesel engine. Energy 35(9): 3628-3639

[13] Gadallah A H, Elshenawy E A, Elzahaby A M, El-Salmawy H A and Bawady A H 2009 Effect of In Cylinder Water Injection Strategies on Performance and Emissions of a Hydrogen Fuelled Direct Injection Engine. SAE Technical Paper Series

[14] Younkins, Wooldridge M S and Boyer B A 2015 Port Injection of Water into a DI Hydrogen Engine. SAE Technical Paper 2015-01-0861

[15] Psota M A, Easley W L, Fort T H and Mellor A M 1997 Water injection effects on NOx emissions for engines utilizing diffusion flame combustion. SAE Technical Papers 106: 1835-1843

[16] Berni F, Breda S, Lugli M and Cantore G 2015 A Numerical Investigation on the Potentials of Water Injection to Increase Knock Resistance and Reduce Fuel Consumption in Highly Downsized GDI Engines. Energy Procedia 81: 826-835

[17] D'Adamo A, Berni F, Breda S, Lugli M, Fontanesi S and Cantore G 2015 A Numerical Investigation on the Potentials of Water Injection as a Fuel Efficiency Enhancer in Highly Downsized GDI Engines. SAE Technical Paper Series

[18] Boretti A 2013 Water injection in directly injected turbocharged spark ignition engines. Appl. Therm. Eng. 52(52): $62-68$ 